\title{
Segmental Abnormalities of White Matter Microstructure in End-Stage Renal Disease Patients: An Automated Fiber Quantification Tractography Study
}

Yuhan Jiang ${ }^{1+}$, Yangyingqiu Liu'1+, Bingbing Gao', Yiwei Che², Liangjie Lin³, Jian Jiang', Peipei Chang', Qingwei Song', Nan Wang ${ }^{4}$, Weiwei Wang ${ }^{1}$ and Yanwei Miao ${ }^{1 *}$

'Department of Radiology, The First Affiliated Hospital of Dalian Medical University, Dalian, China, ${ }^{2}$ Department of Radiology, The Third People's Hospital of Dalian, Dalian, China, ${ }^{3}$ Philips Healthcare China, Beijing, China, ${ }^{4}$ Department of Nephrology, The First Affiliated Hospital of Dalian Medical University, Dalian, China

\section{OPEN ACCESS}

Edited by:

Shantanu H. Joshi,

University of California, Los Angeles,

United States

Reviewed by:

Yan Zhou,

Shanghai Jiao Tong University, China

Niharika Gajawelli,

Children's Hospital of Los Angeles,

United States

*Correspondence:

Yanwei Miao

ywmiao716@163.com

${ }^{\dagger}$ These authors have contributed equally to this work

Specialty section: This article was submitted to Brain Imaging Methods,

a section of the journal

Frontiers in Neuroscience

Received: 27 August 2021 Accepted: 25 October 2021 Published: 06 December 2021

Citation:

Jiang Y, Liu Y, Gao B, Che Y, Lin L, Jiang J, Chang $P$, Song $Q$, Wang N, Wang W and Miao Y (2021) Segmental Abnormalities of White Matter Microstructure in End-Stage Renal Disease Patients: An Automated Fiber Quantification Tractography Study.

Front. Neurosci. 15:765677. doi: 10.3389/fnins.2021.765677
Background and Purpose: End-stage renal disease (ESRD) results in extensive white matter abnormalities, but the specific damage segment cannot be identified. This study aimed to determine the segmental abnormalities of white matter microstructure in ESRD and its relationship with cognitive and renal function indicators.

Methods: Eighteen ESRD patients and 19 healthy controls (HCs) were prospectively recruited. All participants underwent DTI and clinical assessments. Automatic fiber quantification (AFQ) was applied to generate bundle profiles along 16 main white matter tracts. We compared the DTI parameters between groups. Besides, we used partial correlation and multiple linear regression analyses to explore the associations between white matter integrity and cognitive performance as well as renal function indicators.

Results: In the global tract level, compared to HCs, ESRD patients had greater MD, AD, and RD values and lower FA value in several fibers ( $P<0.05$, FDR correction). In the point-wise level, extensive damage existed in specific locations of different fiber tracts, particularly in the left hemisphere $(P<0.05$, FDR correction). Among these tracts, the mean $A D$ values of the left cingulum cingulate correlated negatively with MoCA score. Urea and UA level were independent predictors of the AD value of superior component of the left corticospinal. Besides, urea level was the independent predictors of mean MD value of left anterior thalamic radiation (ATR).

Conclusion: White matter fiber tract damage in ESRD patients may be characterized by abnormalities in its specific location, especially in the left hemisphere. Aberrational specific located fibers were related to cognitive impairment and renal dysfunction.

Keywords: white matter microstructure, end-stage renal disease, diffusion tensor imaging, automated fiber quantification, serum urea, uric acid

Abbreviations: AFQ, automated fiber quantification; AD, axial diffusivity; ATR, anterior thalamic radiation; CST, corticospinal; Cre, Creatinine; ESRD, end stage renal disease; FA, fractional anisotropy; FDR, false discovery rate; IFOF, inferior fronto-occipital fasciculus; ILF, inferior longitudinal fasciculus; MD, mean diffusivity; MoCA, Montreal Cognitive Assessment; RD, radial diffusivity; SLF, superior longitudinal fasciculus; Urea, serum urea; UA, uric acid. 


\section{INTRODUCTION}

Chronic kidney disease (CKD) is a condition characterized by a gradual loss of kidney function. Estimated glomerular filtration rate (eGFR) is an objective indicator for evaluating glomerular filtration capacity and is usually used to reflect renal function. End-stage renal disease (ESRD) is the final stage of chronic kidney disease (CKD), which is defined as an eGFR of less than $15 \mathrm{ml} / \mathrm{min} / 1.73 \mathrm{~m}^{2}$. ESRD patients require renal replacement therapy via maintenance dialysis or kidney transplantation to maintain life (National Kidney Foundation, 2002; Romagnani et al., 2017). CKD induces a series of pathophysiological processes which in turn affect the structural and functional integrity of the brain, which is more pronounced in ESRD patients (Lu et al., 2015). In addition, ESRD patients are often accompanied by cognitive decline. A study has confirmed that ESRD is an independent risk factor for cognitive impairment (O’Lone et al., 2016).

Neuroimaging research has greatly promoted our understanding of the ESRD-related neurological changes. Most of the previous quantitative analyses focused on the abnormal structure of gray or white matter (Zhang et al., 2013; Murea et al., 2015; Meurs et al., 2016). In recent years, more and more studies have confirmed that the integrity of white matter microstructure in ESRD patients has also changed using various analytical methods based on diffusion tensor imaging (DTI), including region of interest (ROI), voxel-based analysis (VBA), and tract-based spatial statistics (TBSS) (Chou et al., 2013, 2019; Kong et al., 2014; Chen et al., 2015; Yin et al., 2018; Liu et al., 2020). However, the location and range of decrease of white matter integrity varied differently across studies. For example, a ROI-based study found that compared with the control group, FA values in all regions were reduced (Hsieh et al., 2009). Results from VBA studies showed FA reduction within the middle cerebellar peduncle, bilateral sagittal stratum, and the genu and splenium of the corpus callosum (Chou et al., 2013). TBSS results have shown that lower FA values within the bilateral corona radiata (Kong et al., 2014; Yin et al., 2018), bilateral inferior fronto-occipital fasciculus (IFOF) (Eldehni et al., 2019), bilateral superior longitudinal fasciculus (SLF) (Zhang et al., 2015; Yin et al., 2018), left anterior thalamic radiation (ATR) (Zhang et al., 2015), the body of the corpus callosum (Drew et al., 2017), and bilateral inferior longitudinal fasciculus (ILF) (Eldehni et al., 2019). In addition, ROI-based analysis lacks consistent standard and largely dependent on the personal evaluation. Because of the differences in the shape of the white matter fiber bundles between subjects, VBA is not accurate enough at the individual level (Wassermann et al., 2011). Although TBSS can be more accurate than VBA, it still fails to provide specific properties for positioning the white matter integrity along each fiber bundle (Bach et al., 2014). Automatic fiber quantification (AFQ) is a new algorithm that can automatically identify the main fiber bundles, allowing researchers to quantify diffusion indicators at anatomically equivalent locations along the fiber tracts (Yeatman et al., 2012). This tract-oriented automatic quantization approach provides more detailed diffusion parameters when mean measurement along each tract is not obvious.

To our best knowledge, this is the first study of applying the AFQ technique to explore potential regional white matter fiber alterations in ESRD patients. We hypothesize that abnormal white matter integrity existed in specific regions of tracts in ESRD patients. We also explored the relationships of DTI metrics with clinical and laboratory characteristics to further investigate the locations of brain structure changes that are associated with certain clinical manifestations.

\section{MATERIALS AND METHODS}

\section{Participants}

This is a prospective study approved by the ethics committee of the first affiliated hospital of our Medical University, and written informed consent was obtained from each participant. The inclusion criteria for ESRD were: (a) confirmed ESRD diagnosis by nephrologist based on the kidney disease outcomes quality initiative (K/DOQI) classification; (b) maintenance hemodialysis (3-4 times per week) for at least 3 months; (c) right-handedness; and (d) age $>18$ years. Exclusion criteria included: (a) diagnosis of psychiatric or neurological disorders (e.g., infarction), traumatic brain injury; (b) recipient of renal transplant or acute renal failure (ARF); and (c) contraindications for MRI examination (e.g., claustrophobia, pacemaker).

Between April 2019 and June 2021, 23 patents diagnosed with ESRD were prospectively enrolled. Patients with poor image quality $(n=3)$ and with claustrophobia $(n=2)$ were excluded. Therefore, 18 ( 10 men; 8 women; mean age, 57.28 years; age range, 33-74) ESRD patients were enrolled in the final analysis. Nineteen healthy controls ( 8 men; 11 women; mean age, 56.05; age range, 34-75; HCs, right-handedness) were recruited with similar gender, age, body mass index (BMI), and education level to the ESRD patients. The exclusion criteria for HCs were traumatic brain injury, mental, and neurological disorders.

\section{Magnetic Resonance Imaging Acquisition}

Magnetic resonance imaging (MRI) scans were performed using a 3.0-T MRI scanner (Ingenia CX, Philips Healthcare, Best, the Netherlands) equipped with a 32-channel phased-array head coil. High-resolution, three-dimensional (3D), T1-weighted (T1W) images were obtained using a multi-shot turbo field echo (MSTFE) sequence with the following scan parameters: echo time $(\mathrm{TE})=3.0 \mathrm{~ms}$, repetition time $(\mathrm{TR})=6.6 \mathrm{~ms}$, flip angle $(\mathrm{FA})=12^{\circ}$, slices $=188$, field of view $(\mathrm{FOV})=256 \times 256 \mathrm{~mm}^{2}$, matrix size $=256 \times 256$, and thickness $=1.0 \mathrm{~mm}$. We obtained the DTI data using a single-shot echo planar imaging (SSEPI) sequence $\left(\mathrm{TE}=92 \mathrm{~ms}\right.$, $\mathrm{TR}=6,000 \mathrm{~ms}, \mathrm{FA}=90^{\circ}$, voxel size $=2 \times 2 \times 2 \mathrm{~mm}^{3}$, FOV $=256 \times 256 \mathrm{~mm}$, matrix size $=128 \times 128,68$ axial slices of $2 \mathrm{~mm}$ thickness to cover the whole brain without gap). Each DTI dataset included 64 noncollinear spatial directions at $b$-value $=1,000 \mathrm{~s} / \mathrm{mm}^{2}$ and one baseline image at $b=0 \mathrm{~s} / \mathrm{mm}^{2}$. 


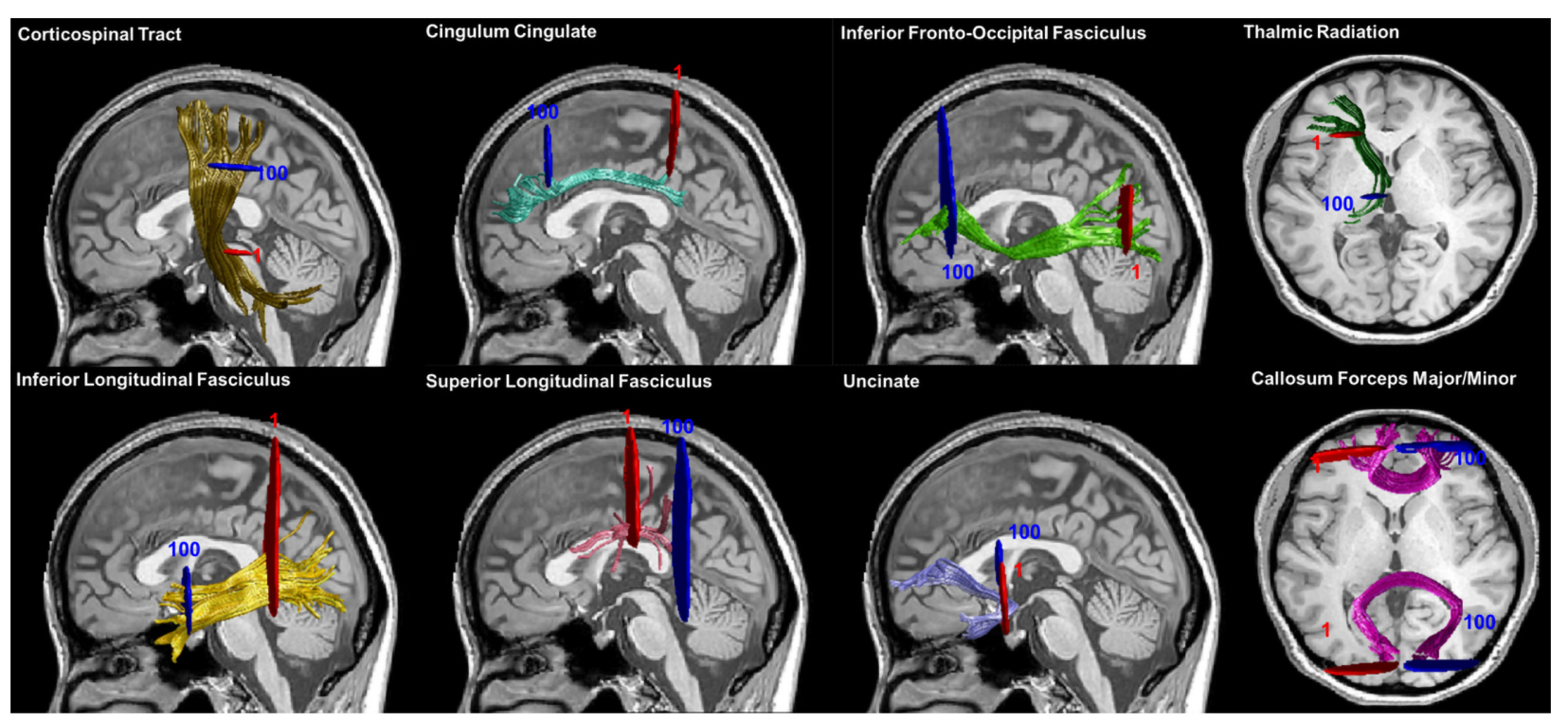

FIGURE 1 | Fiber tracts identification results. Sixteen main fiber tracts were successfully identified by AFQ. Starting and ending waypoint ROls are described in red and blue, respectively. Abbreviation: ROls, regions of interest.

\section{Data Preprocessing}

Diffusion-weighted images were preprocessed by the opensource Vistasoft package version 1.0. ${ }^{1}$ Eddy current-induced distortion correction, motion artifact correction, and skull stripping were all performed using the Functional MRI of the Brain (FMRIB) Software Library (FSL) version 5.0.9 (Smith et al., 2004). A diffusion tensor model at each voxel was fitted using DTIFIT command of FSL to generate fractional anisotropy (FA), mean diffusivity (MD), eigenvalue $(\lambda 1, \lambda 2, \lambda 3)$ maps and a raw T2 image without diffusion weighting (S0). Then the axial $(\mathrm{AD}=\lambda 1)$ and radial $[\mathrm{RD}=(\lambda 2+\lambda 3) / 2]$ diffusivity maps were calculated for each participant. For the $3 \mathrm{D}$ T1W scans, firstly, using "Brain Extraction Tool (BET)," a function of the FSL, to remove non-brain structures. Then, the T1W images were averaged and rotated to align with the anterior commissureposterior commissure (AC-PC) plane using FSL. Finally, the script dtiMakeDt6FromFSL was used to obtain a dt6 MATLAB format file by aligning the T1W image to the S0 image.

\section{Automated Fiber Quantification}

Twenty major fiber tracts of the whole brain were preliminarily identified and we quantified the diffusion metrics along the tracts by applying an open-source MATLAB version of AFQ (Yeatman et al., 2012). The identification procedure included the following procedures: (1) whole-brain deterministic fiber tractography using a streamline tracking algorithm with thresholds of FA $>0.2$ and turning angle $<30^{\circ}$; (2) waypoint ROIbased tract segmentation (Wakana et al., 2007); (3) fiber tracts refinement based on the probabilistic fiber tract maps (Hua et al., 2008); (4) fiber tract cleaning using an iterative procedure

${ }^{1}$ https://github.com/vistalab/vistasoft

${ }^{2}$ http://fsl.fmrib.ox.ac.uk/fsl/ to remove fibers more than four standard deviations (SDs) above the mean fiber length or far from the core of the fiber bundle (Yeatman et al., 2012); and (5) quantification of the DTI metrics of each participant at 100 equidistant nodes along each fiber tract. However, we failed to identify some fibers in large portion of participants due to the strict criterion for tract segmentation, including the bilateral arcuate fasciculus (AF) and bilateral cingulum hippocampus $(\mathrm{CH})$. Therefore, four fiber tracts were excluded from the subsequent analysis. The fully identified 16 fiber tracts were showed in Figure 1.

\section{Neurocognitive Assessments and Clinical/Laboratory Tests}

Among all participants, only 12 ESRD patients and $13 \mathrm{HCs}$ completed cognitive assessment [Beijing revised version Montreal Cognitive Assessment (MoCA)] before MR data acquisition.

All ESRD patients underwent several biochemical tests, including serum urea (urea), uric acid (UA), creatinine (Cre), cystatin $\mathrm{C}$ (Cys C), homocysteine (HCY), low density lipoprotein (LDH), high density lipoprotein (HDL), and triglyceride (TG) levels before MR data acquisition. Participants in the HCs group did not undergo biochemical tests.

\section{Statistical Analyses}

SPSS Statistics V22.0. was used to analyze the demographic characteristics (including age, gender, BMI, and years of education) and cognitive assessment (MoCA score) from the HC individuals and ESRD patients. We used two-sample $t$-tests or Mann-Whitney $U$-tests to compare normally or non-normally distributed variables, 
TABLE 1 | Demographic and clinical characteristics.

\begin{tabular}{|c|c|c|c|c|}
\hline & $\mathrm{HC}$ & ESRD & $\mathbf{t} / \mathbf{Z} / \mathbf{x}^{2}$ & $P$-value \\
\hline Age, years & $56.05 \pm 11.65$ & $57.28 \pm 12.22$ & -0.312 & 0.757 \\
\hline Gender (male/female) & $8 / 11$ & $10 / 8$ & 0.669 & 0.413 \\
\hline Education, years, M(IQR) & $12(12,15)$ & $13(9.75,15)$ & -0.094 & 0.925 \\
\hline $\mathrm{BMI}, \mathrm{kg} / \mathrm{m}^{2}$ & $24.25 \pm 2.42$ & $23.63 \pm 2.78$ & 0.716 & 0.479 \\
\hline Hypertension, $n$ (\%) & $6(31.58)$ & $15(83.33)$ & - & $0.003^{*}$ \\
\hline Diabetes mellitus, $n(\%)$ & $5(27.78)$ & $9(50.00)$ & - & 0.535 \\
\hline History of smoking, $n$ (\%) & $6(31.58)$ & $4(22.22)$ & - & 0.714 \\
\hline History of drinking, $n$ (\%) & $4(21.05)$ & $3(16.67)$ & - & 1.000 \\
\hline MoCA, $n, \mathrm{M}(\mathrm{IQR})$ & $13,26(25,28)$ & $12,23.5(22,24.25)$ & -2.894 & $0.004^{\star}$ \\
\hline Visuospatial/executive, M(IQR) & $5(3,5)$ & $4(3,4)$ & -1.104 & 0.320 \\
\hline Naming, $M(I Q R)$ & $3(3,3)$ & $3(2.75,3)$ & -1.155 & 0.248 \\
\hline Attention, M(IQR) & $6(5,6)$ & $5(4,6)$ & -1.239 & 0.215 \\
\hline Language, M(IQR) & $2(1,2)$ & $2(1,2)$ & -0.237 & 0.812 \\
\hline Abstraction, M(IQR) & $2(2,2)$ & $2(1.75,2)$ & -0.311 & 0.756 \\
\hline Delayed recall, M(IQR) & $4(4,5)$ & $2.5(2,3)$ & -3.056 & $0.002^{*}$ \\
\hline Orientation, M(IQR) & $6(6,6)$ & $6(5.75,6)$ & -1.883 & 0.060 \\
\hline
\end{tabular}

HC, health controls; BMI, body mass index; ESRD, end stage renal disease; $M$, median; $n$, number; IQR, interquartile range.

Values are presented as mean \pm standard deviation (SD), $n$ (\%), or M (IQR).

${ }^{*}$ Represents the statistical difference between the two groups, $P<0.05$.

respectively. Chi-squared $\left(\chi^{2}\right)$ tests or Fisher's exact tests were performed in the comparisons of categorical data. The significant threshold was set at corrected $P<0.05$.

First, we compared the group differences of WM tracts from the level of global tract; average DTI metrics (FA, MD, AD, and $\mathrm{RD}$ ) were calculated by averaging these diffusion values of 100 nodes along each fiber tract and the two-sample $t$-test was performed to determine the differences.

Then, we applied the point-wise analyses based on the "Randomize" command in FSL, controlling gender, age, BMI, and years of education as covariates in the general linear model (GLM). A non-parametric permutation-based statistical analysis (5,000 permutations), with false discovery rate (FDR) procedure ( $q=0.05$ ) for multiple comparisons was utilized to the 1,600 points and only significant differences observed at $\geq 3$ adjacent nodes were reported (Banfi et al., 2019).

Correlation analysis between diffusion properties and MoCA scores as well as laboratory tests was performed using partial Pearson correlation, with age, gender, BMI, and education as covariate. FDR correction was applied to reduce the false-positive errors. We then performed multiple linear regression analysis to find independent predictors for explaining the relationship between laboratory tests and DTI metrics. Statistical tests were two-tailed, and $P<0.05$ was considered statistically significant.

\section{RESULTS}

\section{Demographic and Clinical Characteristics}

The demographic and clinical characteristics of the ESRD patients and HCs are summarized in Table 1. Age, gender,
BMI, and years of education between groups showed no statistical difference $(P>0.05)$. The incidence of hypertension in ESRD patients is higher $(P=0.033)$. Meanwhile, poorer performances on $\operatorname{MoCA}(P=0.018)$ were found in ESRD group, especially in delayed recall $(P=0.005)$.

\section{Group Difference in Global Tract Level and Point-Wise Level}

Fractional Anisotropy

For the whole global tract, compared to HCs, lower mean FA values were in the ESRD patients in the right ATR, right cingulum cingulate (CC), bilateral IFOF, bilateral ILF, and callosum forceps minor $(P<0.05$, FDR correction, Supplementary Table 1 and Figure 2).

In the point-wise comparison between groups, the positions of the fiber bundles that changed significantly were as follows: (a) the anterior and posterior component of the left IFOF (nodes 1-9; nodes 18-22; nodes 52-62; and nodes 95-100); (b) the inferior left corticospinal (CST; nodes 25-37); and (c) the frontal and occipital portion of the callosum forceps minor (nodes 16-26 and nodes 68-81; Figure 3, $P<0.05$, FDR correction).

\section{Mean Diffusivity}

Compared to HC, ESRD group showed significantly increased $\mathrm{MD}$ value in multiple fiber bundles, including the bilateral CST, CC, ILF, IFOF, and ATR, the left SLF and callosum forceps minor $(P<0.05$, FDR correction, Supplementary Table 2 and Figure 4).

In the point-wise comparison of MD values, the significantly altered fiber bundle locations $(P<0.05$, FDR correction) were as follows: (a) the inferior and superior left CST (nodes 

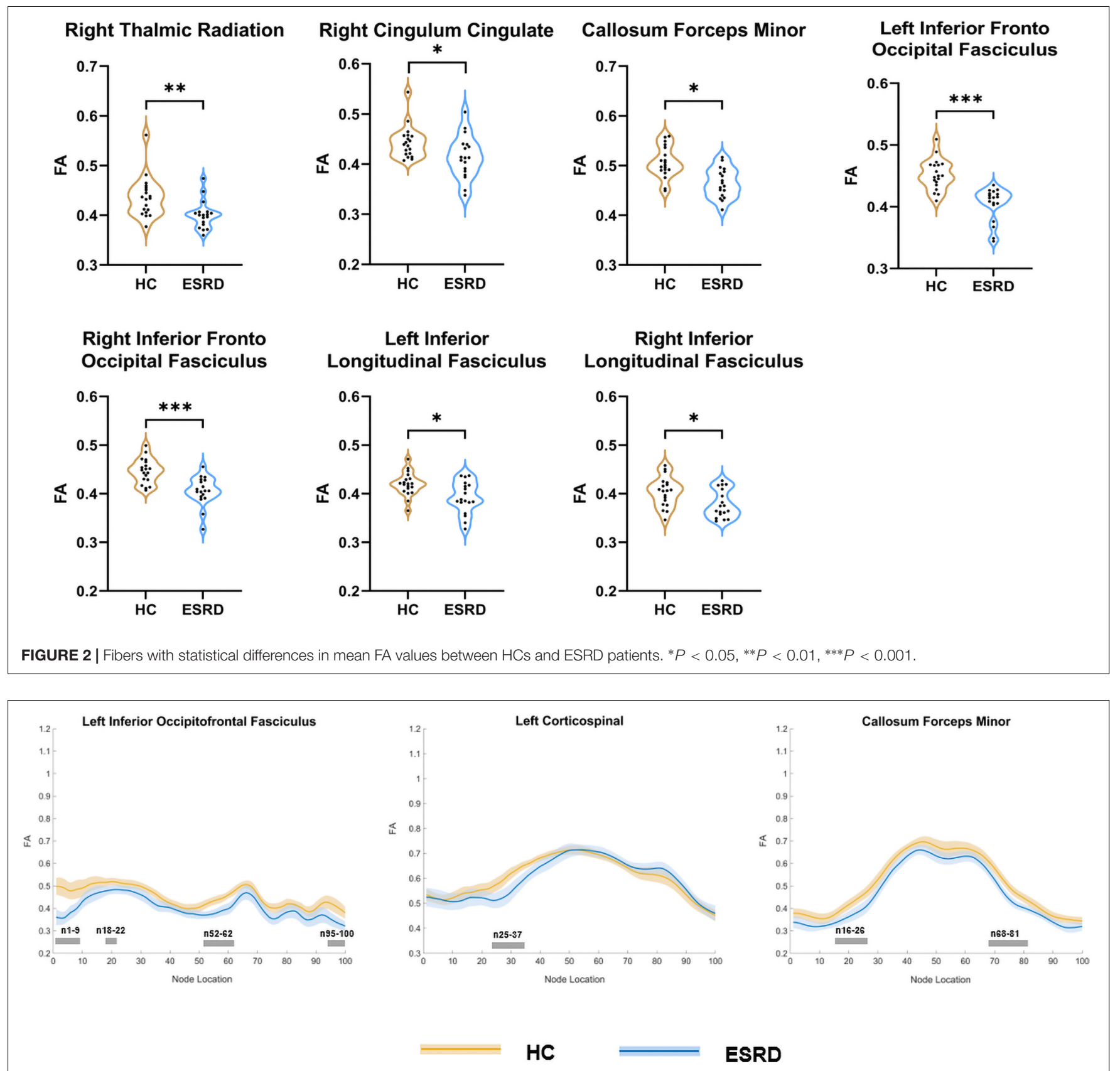

FIGURE 3 | Plots of significantly altered locations in point-wise comparison of FA values between HCs and ESRD $(P<0.05$, FDR correction). The orange line represents the HC group; the blue line represents the ESRD group (solid lines for means and shaded regions for confidence interval). The gray bars at the bottom are the regions of the fiber segment with significant difference between the two groups. ESRD, end stage renal disease; HC, health control; FA, fractional anisotropy.

12-14; nodes 19-39; and nodes 67-100) and right CST (nodes 12-30 and nodes 64-100); (b) the occipital portion of the forceps major (nodes 90-100) and the widespread distribution of the callosum forceps minor (nodes 1-40 and nodes 47-100); (c) the anterior, intermediate, and posterior parts of the left IFOF (nodes 1-8; nodes 34-69; and nodes 88-100); (d) wide distribution of the biliteral ILF (L: nodes 1-76 and nodes 87-93; R: nodes 35-60 and nodes 80-83); (e) the widespread distribution of the left SLF (nodes 1-57 and nodes 74-100); and (f) the occipital lobe component of the right uncinate fasciculus (UF, nodes 10-18; Figure 5).

\section{Axial Diffusivity}

In comparison with the HCs, the ESRD group exhibited significantly greater AD values in the bilateral CST, left CC, and bilateral ILF (FDR correction, Supplementary Table 3 and Supplementary Figure 1). 


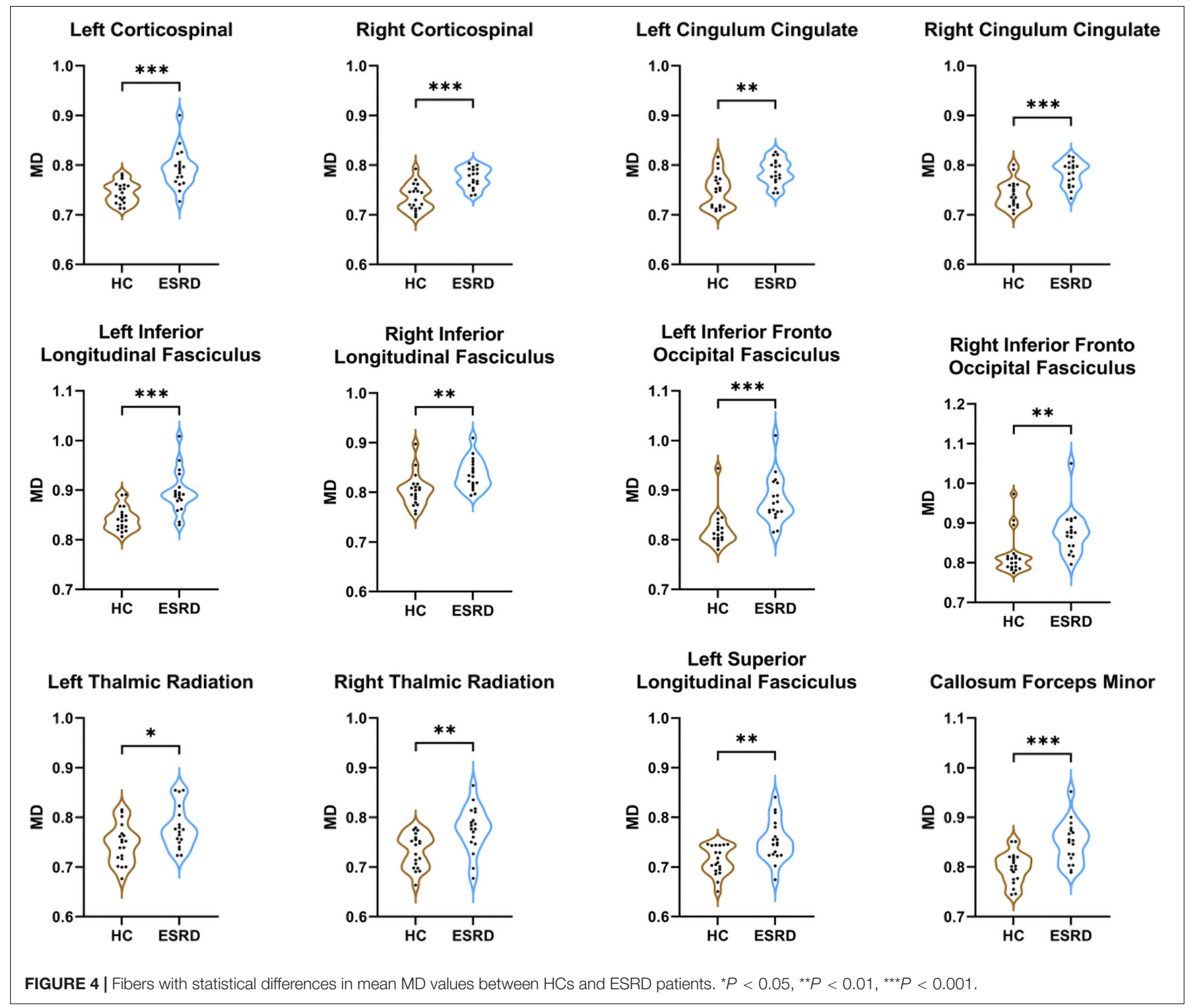

Point-wise comparison showed that the specific location changes of fibers $(P<0.05$, FDR correction) as follows: (a) the superior parts of the left CST (nodes 66-100) and right CST (nodes 67-96) and (b) the posterior component of the left ILF (nodes 64-66; Supplementary Figure 2).

\section{Radial Diffusivity}

In the fiber tract level, the callosum forceps minor, bilateral CST, bilateral CC, bilateral IFOF, bilateral ILF, left SLF, right ATR, and right UF exhibited significant differences between HC and ESRD groups (FDR correction, Supplementary Table 4 and Supplementary Figure 3).

The following fibers had significant changes in the pointwise comparison $(P<0.05$, FDR correction), including (a) the inferior portion of the left CST (nodes 20-40) and right CST (nodes 12-29); (b) the frontal and occipital portion of the forceps major (nodes 14-18 and nodes 80-85) and the entire fiber bundle of the callosum forceps minor (nodes 1-100); (c) the widespread distribution of the left IFOF (nodes 1-9, nodes 19-22, nodes 3244, nodes 49-67, and nodes 87-100); (d) the wide distribution of the left ILF (nodes 1-13, nodes 19-61, and nodes 89-95) and intermediate and posterior parts of the right ILF (nodes 34-60 and nodes 81-83); (e) the anterior part of the left SLF (nodes 427 ); and (f) the frontal component part of the right UF (nodes 94-100; Supplementary Figure 4).

\section{Correlation Analysis}

Correlations Between Diffusion Metrics and Montreal Cognitive Assessment and Biochemical Tests

Among the 12 ESRD patients who completed MoCA assessment, partial correlation analysis between MoCA and diffusion metrics of each fiber cluster where group differences emerged or mean values of fiber tracts were performed. 


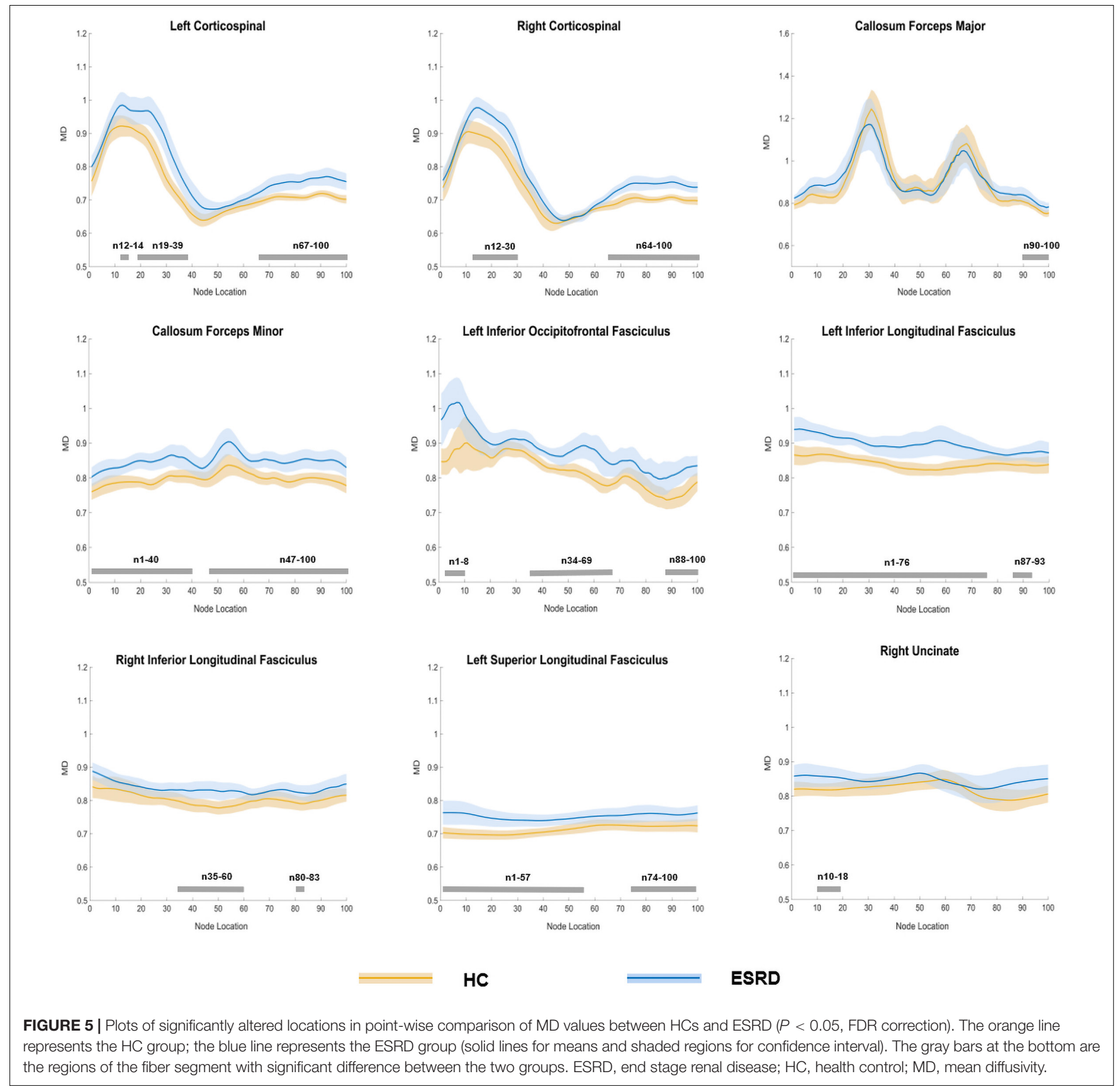

We found highly positive correlation between visuospatial/executive score and the mean $\mathrm{FA}$ values of the right ATR $(r=0.823, P=0.012)$; the mean AD values of the left CC were negatively related to total MoCA score $(r=-0.836$, $P=0.010)$ and visuospatial/executive score $(r=-0.898, P=0.002)$. Significant negative correlations were discovered between the RD values of the occipital component part of the callosum forceps major and naming score (nodes 14-18, $r=-0.748, P=0.033$; Supplementary Figure 5).

See Supplementary Figure 6 for the details of the partial correlation analysis between biochemical tests and diffusion metrics.

\section{Multiple Linear Regression Analysis}

The multiple regression analysis revealed that urea and UA level were independent predictors of the $\mathrm{AD}$ value of superior component of the left CST. Besides, we also found that urea level was the independent predictors of mean MD value of left ATR (Table 2).

\section{DISCUSSION}

In this study, we first investigate the specific alterations of white matter microstructure in patients with ESRD by AFQ 
TABLE 2 | Multiple linear regression analysis.

\begin{tabular}{lcc}
\hline & $\boldsymbol{\beta}$ & $\boldsymbol{P}$ \\
\hline AD value of N66-100 of left CST & & 0.007 \\
Urea & 0.407 & 0.000 \\
UA & 0.311 & \\
Mean MD value of left ATR & & 0.034 \\
Urea & 0.528 &
\end{tabular}

ATR, anterior thalamic radiation; $A D$, axial diffusivity; $M D$, mean diffusivity; CST, corticospinal tract; Urea, serum urea; Cre, creatinine; UA, uric acid.

and further explored the relationships of white matter integrity with MoCA scores and biochemical indicators. We found the following points: (1) ESRD patients had extensive microstructure fragility of white matter fiber bundles, especially on the left side of the hemisphere; (2) changes of white matter microstructure might not only occur along the entire white matter fiber bundle, but also at some specific fiber segments; and (3) the point-wise level analysis was more sensitive than fiber tract level analysis and white matter fiber damage might be associated with clinical symptoms and cognitive function.

There are several mechanisms for the development of cognitive dysfunction related to white matter microstructure fragility in ESRD patients. First, the accumulation of neurotoxins caused by dialysis or uremia can cause central nervous system complications (Smogorzewski, 2001). Second, asymptomatic cerebral infarction, white matter lesions, and microhemorrhage caused by degenerative microvascular disease can cause vascular dementia (Arismendi-Morillo and Fernández-Abreu, 2010). Third, hemodialysis leads to changes in cerebral blood flow, leading to brain tissue and metabolic disorders (Kanai et al., 2001). Fourth, increased oxidative stress and chronic inflammation in ESRD may lead to endothelial and neuronal damage (Bugnicourt et al., 2013).

In our research, we applied four diffusion metrics to evaluate the alterations in the microstructure of white matter fiber bundles. FA and MD reflect the overall directionality and magnitude of water diffusion, respectively (Le Bihan et al., 2001). $\mathrm{RD}$ and $\mathrm{AD}$ denote the extent of diffusion perpendicular and parallel to fiber orientations, respectively (Assaf and Pasternak, 2008). All these metrics are purported to be sensitive to myelin and axon damage. Therefore, by using MD, AD, and $\mathrm{RD}$ values together with the FA value, white matter changes in ESRD patients can be better characterized. In comparison with $\mathrm{HCs}$ at the overall fiber level, ESRD patients exhibited widespread damaged tract profiles, with significant FA reduction in $43.75 \%(7 / 16)$ and $\mathrm{MD}, \mathrm{AD}$, and $\mathrm{RD}$ increase in $75 \%$ $(12 / 16), 31.25 \%(5 / 16)$, and $75 \%(12 / 16)$ of the fiber tracts examined, respectively. And point-wise level analysis allowed us to obtain more accurate damaged segments, especially in $\mathrm{MD}$ and $\mathrm{RD}$. These damage segments were mainly observed on fibers included projection fiber (CST), commissural fibers (forceps minor, forceps major), and association fibers (IFOF, ILF, SLF), especially for the association fibers, which have changed in all the four diffusion metrics. IFOF, as the longest associative bundle, is an important link connecting the frontal, temporal, and occipital lobes (Caverzasi et al., 2014) and plays a major role in the neuromotor function and processing of vison and hearing. Studies have found that the loss of WM integrity in IFOF have been considered a risk factor for Alzheimer's disease, and Alzheimer's disease patients show extensive damage along the IFOF bundle, including the anterior, posterior, and central region associated with memory impairment (Fu et al., 2014; Chen H. F. et al., 2020). Our findings indicated that ESRD patients have extensive damage to the left IFOF, including the anterior, posterior, and central regions, and mainly characterized in the form of decreased FA values and increased MD values, indicating that the microstructure changes of IFOF are mainly myelin damage. ILF is a critical pathway that primarily connects the occipital cortex and temporal lobe. Impaired integrity of ILF and SLF is associated with language comprehension and attention-deficit disorders (Wolfers et al., 2015; Shin et al., 2019). These results are similar to previous TBSS studies on CKD (Yin et al., 2018; Liu et al., 2020), while more detailed localization information of damaged segments of fibers can be determined in our current study based on AFQ analyses. Meanwhile, these results indicated that $\mathrm{MD}$ and $\mathrm{RD}$ values are more sensitive than FA in discovering the extent of white matter microstructure vulnerability, which consistent with previous studies (Amlien and Fjell, 2014; Jin et al., 2017; Chen H. F. et al., 2020). In addition, this study found that almost all segments of forceps minor had abnormal white matter microstructures, and with the accumulation of urea, the microstructure of nodes 16-26 in the forceps minor tended to damage. The corpus callosum is composed of white matter tracts that connect the left and right cerebral hemispheres and is the main commissural region of the brain. And the forceps minor is the fiber that connects the lateral and medial regions of the bilateral frontal lobes, which is involved in processing speed (Kerchner et al., 2012). Patients with ESRD can experience cognitive changes in different aspects, and the decline in executive function is the most obvious (Berger et al., 2016). Based on the point of view, this study found that with the decline of renal function, the microstructure of the frontal part of the forceps minor may be damaged, which may lead to impaired cognitive function. Unfortunately, our study did not carry out tests related to executive function, and further research is needed.

Besides, our study found that most of the damaged fiber bundles were in the left hemisphere, suggesting that the white matter vulnerability may have hemispheric heterogeneity. Previous study on microstructure damage in Alzheimer's disease has also found heterogeneity in brain structure changes between hemispheres (Chen H. et al., 2020). At present, studies have shown that patients with $\mathrm{CKD}$ are more prone to result in cognitive decline and Alzheimer's disease (Etgen et al., 2012; Zhang et al., 2020). Therefore, we speculate that these changes in brain white matter structure of ESRD patients may be related to the occurrence and development of Alzheimer's disease.

In addition, this study found that changes in the diffusion metrics of ATR were related to the decline in cognitive level, especially visuospatial/executive. The ATR, which connects the mediodorsal and anterior thalamic nuclei (ATN) with the frontal cortex and the anterior cingulate cortex, can process incoming information from the hippocampus (related to cognitive functions) (Setiadi et al., 2021). Therefore, the decreased integrity 
of ATR may be associated with decreased cognitive functions. Different from the hemispheric heterogeneity reflected by the comparison between groups, we found that the cognitive-related ATR white matter damage were mainly located in the right hemisphere, which may be related to the priority of damage first appearing in the dominant hemisphere. Besides, a study related to reduced attention of young bilingual adults showed that the integrity of the white matter fibers connecting the anterior cingulate cortex and the frontal regions was decreased, and this manifestation only exists in the right hemisphere (Mamiya et al., 2018). Similarly, Huang et al. (2020) also reported that the right ATR was associated with executive function in cerebral small vessel disease patients.

Excitingly, this work found that specific white matter segments were closely associated with specific laboratory test results, such as urea, UA, and Cre. In particular, through multiple linear regression analysis, these specific segments corresponding to specific renal function indexes were determined. Put simply, renal dysfunction in ESRD patients may mediate the corresponding specific white matter segment abnormalities. In this study, we found that with the increase of urea level, mean $\mathrm{AD}$ value of the left ATR and nodes of 66-100 in the left CST tended to be damaged. Besides, our study showed positive correlations of urea level with the increased MD in the left ATR. Our interpretation of this result is that impaired renal function can result in the continuous accumulation of metabolites, which in turn leads to demyelination and axonal damage (Mogi and Horiuchi, 2011; Lu et al., 2015). Previous TBSS studies on ESRD have also demonstrated that serum creatinine is related to white matter integrity (Kim et al., 2011; Yin et al., 2018).

Despite the advantages of this method, we should not ignore some limitations of this study. First, our prospective study was conducted on a relatively small sample, and further investigations on relatively large population are still needed. Second, due to the threshold setting in fiber tracking, some fibers such as the $\mathrm{CH}$ and $\mathrm{AF}$ were adjacent to the gray matter, which makes them impossible to be tracked. Third, in this study, we only focused on patients with ESRD, and in the future, patients with early CKD should be enrolled to explore the correlation between disease progression and white matter microstructure changes. Fourth, due to participant compliance, not all participants had completed the cognitive assessments, which may have a certain impact on the relevant results.

\section{CONCLUSION}

In conclusion, this study identified specific abnormalities in major white matter fibers in ESRD using a tract profile

\section{REFERENCES}

Amlien, I. K., and Fjell, A. M. (2014). Diffusion tensor imaging of white matter degeneration in Alzheimer's disease and mild cognitive impairment. Neuroscience 276, 206-215. doi: 10.1016/j.neuroscience.2014.02.017

Arismendi-Morillo, G., and Fernández-Abreu, M. (2010). Ultrastructural cutaneous microvascular pathology of young adults aged up to 50 approach. Besides, the results of our study indicates that the accumulation of serum urea and uric acid may lead to vulnerable white matter microstructure. We believe that the findings of this work will enhance our understanding of white matter abnormalities in ESRD.

\section{DATA AVAILABILITY STATEMENT}

The raw data supporting the conclusions of this article will be made available by the authors, without undue reservation.

\section{ETHICS STATEMENT}

This study was approved by the Ethics Committee of the First Affiliated Hospital of Dalian Medical University. The patients/participants provided their written informed consent to participate in this study. Written informed consent was obtained from the individual(s) for the publication of any potentially identifiable images or data included in this article.

\section{AUTHOR CONTRIBUTIONS}

YJ and YL: guarantor of integrity of entire study. YJ, YL, BG, YC, JJ, and PC: literature research. YJ, YL, BG, YC, JJ, PC, QS, LL, NW, WW, and YM: clinical studies. YJ, YL and YM: experimental studies. YJ, YL, and JJ: statistical analysis. YJ, YL, LL, and YM: manuscript editing. All authors: study concepts, study design, data acquisition, data analysis, interpretation, manuscript drafting, manuscript revision for important intellectual content, approval of final version of submitted manuscript, agrees to ensure any questions related to the work are appropriately resolved.

\section{FUNDING}

This study was supported by the National Natural Science Foundation of China (Nos. 81671646 and 81801657).

\section{SUPPLEMENTARY MATERIAL}

The Supplementary Material for this article can be found online at: https://www.frontiersin.org/articles/10.3389/fnins. 2021.765677/full\#supplementary-material

years with chronic kidney disease and vascular cognitive impairment. Ultrastruct. Pathol. 34, 214-218. doi: 10.3109/019131210037 43690

Assaf, Y., and Pasternak, O. (2008). Diffusion tensor imaging (DTI)-based white matter mapping in brain research: a review. J. Mol. Neurosci. 34, 51-61. doi: 10.1007/s12031-0070029-0 
Bach, M., Laun, F. B., Leemans, A., Tax, C. M., Biessels, G. J., Stieltjes, B., et al. (2014). Methodological considerations on tract-based spatial statistics (TBSS). Neuroimage 100, 358-369.

Banfi, C., Koschutnig, K., Moll, K., Schulte-Körne, G., Fink, A., and Landerl, K. (2019). White matter alterations and tract lateralization in children with dyslexia and isolated spelling deficits. Hum. Brain Mapp. 40, 765-776. doi: 10.1002/hbm. 24410

Berger, I., Wu, S., Masson, P., Kelly, P. J., Duthie, F. A., Whiteley, W., et al. (2016). Cognition in chronic kidney disease: a systematic review and meta-analysis. BMC Med. 14:206. doi: 10.1186/s12916-016-0745-9

Bugnicourt, J. M., Godefroy, O., Chillon, J. M., Choukroun, G., and Massy, Z. A. (2013). Cognitive disorders and dementia in CKD: the neglected kidney-brain axis. J. Am. Soc. Nephrol. 24, 353-363. doi: 10.1681/ASN.2012050536

Caverzasi, E., Papinutto, N., Amirbekian, B., Berger, M. S., and Henry, R. G. (2014). Q-ball of inferior fronto-occipital fasciculus and beyond. PLoS One 9:e100274. doi: 10.1371/journal.pone.0100274

Chen, H., Sheng, X., Qin, R., Luo, C., Li, M., Liu, R., et al. (2020). Aberrant white matter microstructure as a potential diagnostic marker in alzheimer's disease by automated fiber quantification. Front. Neurosci. 14:570123. doi: 10.3389/fnins. 2020.570123

Chen, H. F., Huang, L. L., Li, H. Y., Qian, Y., Yang, D., Qing, Z., et al. (2020). Microstructural disruption of the right inferior fronto-occipital and inferior longitudinal fasciculus contributes to WMH-related cognitive impairment. CNS Neurosci. Ther. 26, 576-588. doi: 10.1111/cns.13283

Chen, H. J., Zhang, L. J., and Lu, G. M. (2015). Multimodality MRI findings in patients with end-stage renal disease. Biomed. Res. Int. 2015:697402.

Chou, M. C., Hsieh, T. J., Lin, Y. L., Hsieh, Y. T., Li, W. Z., Chang, J. M., et al. (2013). Widespread white matter alterations in patients with end-stage renal disease: a voxelwise diffusion tensor imaging study. AJNR Am. J. Neuroradiol. 34, 1945-1951. doi: 10.3174/ajnr.a3511

Chou, M. C., Ko, C. H., Hsieh, T. J., Chang, J. M., and Chung, W. S. (2019). A preliminary report of longitudinal white matter alterations in patients with end-stage renal disease: a three-year diffusion tensor imaging study. PLoS One 14:e0215942. doi: 10.1371/journal.pone.0215942

Drew, D. A., Koo, B. B., Bhadelia, R., Weiner, D. E., Duncan, S., la Garza, M. M., et al. (2017). White matter damage in maintenance hemodialysis patients: a diffusion tensor imaging study. BMC Nephrol. 18:213. doi: 10.1186/s12882017-0628-0

Eldehni, M. T., Odudu, A., and McIntyre, C. W. (2019). Brain white matter microstructure in end-stage kidney disease, cognitive impairment, and circulatory stress. Hemodial. Int. 23, 356-365. doi: 10.1111/hdi.12754

Etgen, T., Chonchol, M., Förstl, H., and Sander, D. (2012). Chronic kidney disease and cognitive impairment: a systematic review and meta-analysis. Am. J. Nephrol. 35, 474-482. doi: 10.1159/000338135

Fu, J. L., Liu, Y., Li, Y. M., Chang, C., and Li, W. B. (2014). Use of diffusion tensor imaging for evaluating changes in the microstructural integrity of white matter over 3 years in patients with amnesic-type mild cognitive impairment converting to Alzheimer's disease. J. Neuroimaging 24, 343-348. doi: 10.1111/ jon. 12061

Hsieh, T. J., Chang, J. M., Chuang, H. Y., Ko, C. H., Hsieh, M. L., Liu, G. C., et al. (2009). End-stage renal disease: in vivo diffusion-tensor imaging of silent white matter damage. Radiology 252, 518-525. doi: 10.1148/radiol.2523080484

Hua, K., Zhang, J., Wakana, S., Jiang, H., Li, X., Reich, D. S., et al. (2008). Tract probability maps in stereotaxic spaces: analyses of white matter anatomy and tract-specific quantification. Neuroimage 39, 336-347. doi: 10.1016/j. neuroimage.2007.07.053

Huang, L., Chen, X., Sun, W., Chen, H., Ye, Q., Yang, D., et al. (2020). Early segmental white matter fascicle microstructural damage predicts the corresponding cognitive domain impairment in cerebral small vessel disease patients by automated fiber quantification. Front. Aging Neurosci. 12:598242. doi: 10.3389/fnagi.2020.598242

Jin, Y., Huang, C., Daianu, M., Zhan, L., Dennis, E. L., Reid, R. I., et al. (2017). 3D tract-specific local and global analysis of white matter integrity in Alzheimer's disease. Hum. Brain Mapp. 38, 1191-1207. doi: 10.1002/hbm.23448

Kanai, H., Hirakata, H., Nakane, H., Fujii, K., Hirakata, E., Ibayashi, S., et al. (2001). Depressed cerebral oxygen metabolism in patients with chronic renal failure: a positron emission tomography study. Am. J. Kidney Dis. 38, S129-S133. doi: 10.1053/ajkd.2001.27421
Kerchner, G. A., Racine, C. A., Hale, S., Wilheim, R., Laluz, V., Miller, B. L., et al. (2012). Cognitive processing speed in older adults: relationship with white matter integrity. PLoS One 7:e50425. doi: 10.1371/journal.pone.005 0425

Kim, H. S., Park, J. W., Bai, D. S., Jeong, J. Y., Hong, J. H., Son, S. M., et al. (2011). Diffusion tensor imaging findings in neurologically asymptomatic patients with end stage renal disease. Neurorehabilitation 29, 111-116. doi: 10.3233/nre2011-0684

Kong, X., Wen, J. Q., Qi, R. F., Luo, S., Zhong, J. H., Chen, H. J., et al. (2014). Diffuse interstitial brain edema in patients with end-stage renal disease undergoing hemodialysis: a tract-based spatial statistics study. Medicine 93:e313. doi: 10. 1097/MD.0000000000000313

Le Bihan, D., Mangin, J. F., Poupon, C., Clark, C. A., Pappata, S., Molko, N., et al. (2001). Diffusion tensor imaging: concepts and applications. J. Magn. Reson. Imaging 13, 534-546. doi: 10.1002/jmri.1076

Liu, M., Wu, Y., Wu, X., Ma, X., Yin, Y., Fang, H., et al. (2020). White matter microstructure changes and cognitive impairment in the progression of chronic kidney disease. Front. Neurosci. 14:559117. doi: 10.3389/fnins.2020.559117

Lu, R., Kiernan, M. C., Murray, A., Rosner, M. H., and Ronco, C. (2015). Kidneybrain crosstalk in the acute and chronic setting. Nat. Rev. Nephrol. 11, 707-719. doi: 10.1038/nrneph.2015.131

Mamiya, P. C., Richards, T. L., and Kuhl, P. K. (2018). Right forceps minor and anterior thalamic radiation predict executive function skills in young bilingual adults. Front. Psychol. 9:118. doi: 10.3389/fpsyg.2018.00118

Meurs, M., Roest, A. M., Groenewold, N. A., Franssen, C. F., Westerhuis, R., Kloppenburg, W. D., et al. (2016). Gray matter volume and white matter lesions in chronic kidney disease: exploring the association with depressive symptoms. Gen. Hosp. Psychiatry 40, 18-24. doi: 10.1016/j.genhosppsych.2016.02.005

Mogi, M., and Horiuchi, M. (2011). Clinical interaction between brain and kidney in small vessel disease. Cardiol. Res. Pract. 2011:306189.

Murea, M., Hsu, F. C., Cox, A. J., Hugenschmidt, C. E., Xu, J., Adams, J. N., et al. (2015). Freedman, Structural and functional assessment of the brain in European Americans with mild-to-moderate kidney disease: Diabetes Heart Study-MIND. Nephrol. Dial. Transplant. 30, 1322-1329. doi: 10.1093/ndt/ gfv030

National Kidney Foundation (2002). K/DOQI clinical practice guidelines for chronic kidney disease: evaluation, classification, and stratification. Am. J. Kidney Dis. 39, S1-S266.

O’Lone, E., Connors, M., Masson, P., Wu, S., Kelly, P. J., Gillespie, D., et al. (2016). Cognition in people with end-stage kidney disease treated with hemodialysis: a systematic review and meta-analysis. Am. J. Kidney Dis. 67, 925-935. doi: 10.1053/j.ajkd.2015.12.028

Romagnani, P., Remuzzi, G., Glassock, R., Levin, A., Jager, K. J., Tonelli, M., et al. (2017). Chronic kidney disease. Nat. Rev. Dis. Primers 3:17088.

Setiadi, T. M., Martens, S., Opmeer, E. M., Marsman, J. C., Tumati, S., Reesink, F. E., et al. (2021). Widespread white matter aberration is associated with the severity of apathy in amnestic mild cognitive impairment: tract-based spatial statistics analysis. Neuroimage Clin. 29:102567. doi: 10.1016/j.nicl.2021.102567

Shin, J., Rowley, J., Chowdhury, R., Jolicoeur, P., Klein, D., Grova, C., et al. (2019). Inferior longitudinal fasciculus' role in visual processing and language comprehension: a combined MEG-DTI Study. Front. Neurosci. 13:875. doi: 10.3389/fnins.2019.00875

Smith, S. M., Jenkinson, M., Woolrich, M. W., Beckmann, C. F., Behrens, T. E., Johansen-Berg, H., et al. (2004). Advances in functional and structural MR image analysis and implementation as FSL. Neuroimage 23 Suppl. 1, S208-S219. doi: 10.1016/j.neuroimage.2004.07.051

Smogorzewski, M. J. (2001). Central nervous dysfunction in uremia. Am. J. Kidney Dis. 38, S122-S128.

Wakana, S., Caprihan, A., Panzenboeck, M. M., Fallon, J. H., Perry, M., Gollub, R. L., et al. (2007). Reproducibility of quantitative tractography methods applied to cerebral white matter. Neuroimage 36, 630-644. doi: 10.1016/j.neuroimage. 2007.02.049

Wassermann, D., Rathi, Y., Bouix, S., Kubicki, M., Kikinis, R., Shenton, M., et al. (2011). White matter bundle registration and population analysis based on Gaussian processes. Inf. Process. Med. Imaging 22, 320-332. doi: 10.1007/9783-642-22092-0_27

Wolfers, T., Onnink, A. M., Zwiers, M. P., Arias-Vasquez, A., Hoogman, M., Mostert, J. C., et al. (2015). Lower white matter microstructure in the superior 
longitudinal fasciculus is associated with increased response time variability in adults with attention-deficit/ hyperactivity disorder. J. Psychiatry Neurosci. 40, 344-351. doi: 10.1503/jpn.140154

Yeatman, J. D., Dougherty, R. F., Myall, N. J., Wandell, B. A., and Feldman, H. M. (2012). Tract profiles of white matter properties: automating fiber-tract quantification. PLoS One 7:e49790. doi: 10.1371/journal.pone.0049790

Yin, Y., Li, M., Li, C., Ma, X., Yan, J., Wang, T., et al. (2018). Reduced white matter integrity with cognitive impairments in end stage renal disease. Front Psychiatry 9:143. doi: 10.3389/fpsyt.2018.00143

Zhang, C. Y., He, F. F., Su, H., Zhang, C., and Meng, X. F. (2020). Association between chronic kidney disease and Alzheimer's disease: an update. Metab. Brain Dis. 35, 883-894. doi: 10.1007/s11011-020-00561-y

Zhang, L. J., Wen, J., Ni, L., Zhong, J., Liang, X., Zheng, G., et al. (2013). Predominant gray matter volume loss in patients with end-stage renal disease: a voxel-based morphometry study. Metab. Brain Dis. 28, 647-654. doi: 10.1007/ s11011-013-9438-7

Zhang, R., Liu, K., Yang, L., Zhou, T., Qian, S., Li, B., et al. (2015). Reduced white matter integrity and cognitive deficits in maintenance hemodialysis ESRD patients: a diffusion-tensor study. Eur. Radiol. 25, 661-668. doi: 10.1007/ s00330-014-3466-5
Conflict of Interest: LL was employed by the company Philips Healthcare China.

The remaining authors declare that the research was conducted in the absence of any commercial or financial relationships that could be construed as a potential conflict of interest.

Publisher's Note: All claims expressed in this article are solely those of the authors and do not necessarily represent those of their affiliated organizations, or those of the publisher, the editors and the reviewers. Any product that may be evaluated in this article, or claim that may be made by its manufacturer, is not guaranteed or endorsed by the publisher.

Copyright (C) 2021 Jiang, Liu, Gao, Che, Lin, Jiang, Chang, Song, Wang, Wang and Miao. This is an open-access article distributed under the terms of the Creative Commons Attribution License (CC BY). The use, distribution or reproduction in other forums is permitted, provided the original author(s) and the copyright owner(s) are credited and that the original publication in this journal is cited, in accordance with accepted academic practice. No use, distribution or reproduction is permitted which does not comply with these terms. 